\title{
Interactive comment on "Impacts of spatial resolutions on projected changes in precipitation extremes: from site- to grid-scales" by Jianfeng Li et al.
}

Jianfeng Li et al.

jianfengli@hkbu.edu.hk

Received and published: 14 August 2017

We appreciate the reviewer's comments and suggestions. We think the points raised by the reviewer have been well known and discussed in others' and our previous studies. Our analysis of the current study also has well considered these points and our conclusions do not contradict the reviewer's concerns. More importantly, to the best of our understanding on his/her comments and reference, we think the questions of our manuscript tries to answer are different from the reviewer's concerns in nature. Below are our point-by-point responses to clarify how our analysis supports the reviewer's comments and how our objectives are different from the reviewer's concerns. 
(1) Reviewer: I thought the current conclusion did not make sense due to GCMs with coarse resolutions showed bad performance in simulating the extremes.

Reply: Our comparisons of extremes estimated from GCMs at grid resolutions of 2.50x2.50 against observation shown in Figs. 4-7 indicate that GCMs with coarse resolutions are not very good at representing extremes, showing that we have fully considered this factor. In fact, our conclusion on insensitivity of pfut-phis (absolute value of an extreme in a future period minus that in a historical period) to spatial resolutions are developed based on the analysis on observational data (as shown in Section 4.1), which was often used as a reference in previous studies. In that part, we interpolated the daily precipitation of station-based observations into precipitation of grid cells with various spatial resolutions and hence estimate pfut-phis based on daily precipitation with various spatial resolutions. We found that pfut-phis estimated at different resolutions change marginally. The design of this experiment was based on the procedure used by Chen and Knutson (2007) published in Journal of Climate, in which sensitivity of $p$ (absolute value of an extreme) to spatial resolutions was discussed. In other words, the validity of the conclusion does not rely on GCM performances. Therefore, it will be appreciated if the reviewer provides more details on the statement "I thought the current conclusion did not make sense due to GCMs with coarse resolutions showed bad performance in simulating the extremes", so as we can better improve our manuscript.

(2) Reviewer: The statistic was not appropriate in western China because lack of observations.

Reply: We explicitly indicated the lack of observations in western China in Line 3-5 Page 4 in the manuscript, showing that we have considered this factor in the observational data. In the additional analysis of this response (point 7), the impacts of the varying density of stations in different regions on our analysis are further discussed based on the updated dataset with 2,088 stations. In our analysis, the grid cells with various resolutions are constructed centered on each of the stations. The numbers of stations covered by grid cells at various resolutions are shown in Fig. R1b below for

Printer-friendly version

Discussion paper

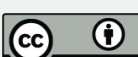


the updated 2,088 stations. The mean of the numbers of stations are around 20 and 40 for $20 \times 20$ and $40 \times 40$ resolutions, respectively, indicating that the numbers of stations are sufficient to estimate the values of grid cells. At the same time, the range of the 10th and 90th percentile of the numbers stations reflects the influence of densities of stations in different areas. This paper aims to generalize the aggregated conditions of sensitivity to spatial resolutions over a vast area, rather than to estimate extremes in a specific region. Therefore, the high density of stations in eastern China can balance the impacts of low density in western China. Fig. R1b will be added in the revised manuscript for readers to better understand the uncertainties.

(3) Reviewer: So this study still focused on the GCMs' resolution impact on climate projection was not a new insight, even combined with statistic downscaling.

Reply: We need to clarify that the focus of the study is impacts of spatial resolutions in general (NOT just GCMs, but also different types of datasets, such as reanalysis and observations, at different resolutions) on changes in extremes (here we only focus on pfut-phis.) based on spatial interpolation. In fact, we mainly used observational data to derive our conclusions (see our reply to point 1). Furthermore, as discussed in our introduction in L25 P2 - P30 L3, spatial resolution impact on climate projection is a popular topic and at the same time very controversial, because many scientific and technical details are involved, for example, dynamical downscaling or statistical downscaling (e.g. Fowler et al., 2007), usages of different RCMs or different statistics for downscaling (e.g. Maraun et al., 2010), orders of interpolating daily precipitation from original resolutions first then estimating extremes vs estimating extremes at original resolutions first then interpolating the extremes (e.g. Chen and Knutson, 2007), comparison of extremes at different resolutions vs at an uniform resolution (e.g. Hu et al., 2016), etc. Different choices of these techniques may lead to very different results. Therefore, although this issue is not new, it still needs more studies to improve our understanding.

This study focuses on the problems in comparing extremes estimated from daily pre-

Printer-friendly version

Discussion paper 
cipitation at different spatial resolutions, rather than simply studying statistical downscaling. We estimate changes in extremes derived from daily precipitation of different datasets, e.g. GCMs, interpolated datasets, etc., at their raw resolutions, and then quantify to what extent changes in extremes at different resolutions are comparable. This study aims to address the gap of the previous studies which have shown sensitivity of magnitudes of extremes (i.e. p) derived from daily precipitation at various resolutions. Here we (a) quantify the sensitivity of pfut-phis derived from daily precipitation in datasets at various spatial resolutions and estimate (b) how this factor affects the comparison of extremes estimated from datasets at different resolutions. As we know, a large number of previous studies compared extremes at various resolutions based on statistical downscaling, interpolation, or direct comparison at various resolutions (e.g. Maraun et al., 2010; Hu et al., 2016). To the best of our knowledge on different types of solutions on comparing extremes at various resolutions, the scientific community does not reach consensus on which solution is better than the others. We expect there will be more studies on changes in extremes based on different methods. Therefore, this study is urgently needed and has certain scientific and practical merits by improving our understanding in impacts of resolutions on estimating changes in extremes.

(4) Reviewer: Here, the statistic downscaling could NOT improve the GCMs resolution too much because of the observations were very limited derived from 509 stations.

Reply: To address this concern, we have updated our dataset to include daily precipitation of 2,088 stations across China with good quality control in this response. We conduct preliminary analysis based on these 2,088 and find out that the conclusions are still the same as those derived from the 509 stations (see additional analysis in point 7 ). In the future revised manuscript, we will update all results with the 2,088 stations. We think these 2,088 stations can address the reviewer's concern.

(5) Reviewer: Some studies have already verified the finer resolution through dynamic downscaling could improve the model performance in terms of precipitation and the

Printer-friendly version

Discussion paper

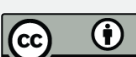


other elements, especially captured the extremes event induced by mesoscale system. And their results also showed different resolutions had great impact to modeling climate change (e.g. Gao et al. 2012 Climate Research).

Reply: We totally agree that models implemented and run in a finer resolution can better represent the precipitation and extremes, because models implemented and parameterized in a finer resolution can better describe the local and regional characteristics and processes, such as the parameteriazation of mirco-physics, clouds formation, and land surface.

However, the question the study tries to discuss is to assess the relative changes in pfut-phis ESTIMATED from daily precipitation at various spatial resolutions based on spatial interpolation and statistical downscaling. In other words, we already have a number of datasets at different spatial resolutions (although we only consider interpolated observations and GCMs, these can be GCM outputs, reanalysis datasets, interpolated products, remote sensing products, etc. in a more general perspective), and then changes in extremes are estimated based on daily precipitation of these datasets. Afterwards, we discuss how differences are pfut-phis estimated at various spatial resolutions. The question is important to understand how comparable are changes in extremes estimated by daily precipitation interpolated to different resolutions for different purposes, given that a large number of previous studies estimating extremes based on interpolation and statistical downscaling. The design of the experiments follows Chen and Knutson (2007) published in Journal of Climate, in which the impacts of spatial resolutions on estimation of the absolute values of extremes were examined. The conclusions of our study agree with the findings of Chen and Knutson (2007) that spatial resolutions have considerable impacts on estimating the absolute values in extremes (i.e. p), e.g. extremes are found to be smaller estimated at coarser resolutions. Building up on this understanding, our conclusions further suggest that the changes of extremes between two periods, i.e. pfut-phis (absolute value of an extreme in a future period minus that in a historical period), have relatively less sensitivity to spatial

Printer-friendly version

Discussion paper 
resolutions compared to the absolute values.

(6) Reviewer: So I suggest the contributors to turn to the GCM-RCM through dynamics downscaling and see if the spatial resolutions have impact on projected changes.

Reply: As far as we know, we hardly see any consensus of scientific community that dynamical downscaling is better than statistical downscaling and vice versus. In fact, as shown in our discussion, these methods have their own advantages and disadvantages (L10-L30 P3). We agree that the impacts of spatial resolutions based on GCM-RCM dynamical downscaling is a very important topic, but our study focuses on impacts of resolutions on changes in extremes by interpolating a larger number of observations into different resolutions. Given many previous studies were based on interpolation and statistical downscaling, we believe the angle and way that we analyze the sensitivity of pfut-phis to spatial resolutions is necessary to improve our understanding of impacts of resolutions on changes in extremes.

\section{(7) Authors: Additional analysis based on the 2,088 stations across China}

To address the concern on the number of stations of observed daily precipitation, we here in this response have collected daily precipitation of more than 2,000 stations of 1961-2005 across China. After quality control, 2,088 stations are selected as shown in Fig. R1a. Similar to the 509 dataset we used previously, the stations are located more in the eastern China and less in the western China. Followed the procedure introduced in the manuscript (L25 P5 - L5 P6), datasets of precipitation extremes at various spatial resolutions from the site scale to the $40 \times 40$ gridded scale are constructed by aggregating daily precipitation of stations covered by grid cells centered at each of the meteorological stations. The relative changes of extremes between 1986-2005 and 1961-1985, i.e. p1986-2005-p1961-1985, are estimated from grid cells at various resolutions. The averaged numbers of stations covered by grid cells are about 20 and 60 at 2ox2o and 40x4o resolutions, respectively (Fig. R1b). The 10th and 90th percentile at $20 \times 20$ the resolution are 5 to 40 , respectively, which are due to the spatially varying 
density of stations in different parts of China. The averaged p1986-2005-p1961-1985 and p1961-1985 across stations from the 2,088 and 509 datasets are compared in Fig. R2 (only CDD and SDII are shown in this response). It is observed that the changes in p1986-2005-p1961-1985 estimated by grid cells at various resolutions are much smaller compared to those in the magnitudes of extremes, i.e. p1961-1985, in both datasets of 2,088 and 509 stations. To better illustrate the sensitivity analysis, an example is shown in Fig. R3. Fig. R3a shows the grid cells with $20 \times 20$ and $40 \times 40$ resolutions centered at a randomly selected station. The number of stations covered by the constructed grid cell increases as the size of the grid cell increases (Fig. R3b). In the typical 2ox2o resolutions of GCMs, about 60 stations have been covered by the 2ox2o grid cell. These 60 stations are aggregated to generate the CDD1961-1985 and CDD1986-2005-CDD1961-1985 of the 20x20 grid cell. The procedure is repeated to estimate extremes with spatial resolutions from the site to $40 \times 40$, and the values of various resolutions are plotted in Fig. 3c. Again, in this example, we can observe that the magnitude of CDD decreases considerably from 62 days to 53 days as grid cell size increases to $40 \times 40$, while the change of CDD fluctuates from -3 to -6 days in these grid cell sizes. Therefore, the changes in extremes are less sensitive to spatial resolutions compared to the magnitudes.

We will complete a more comprehensive analysis based on the 2,088 stations in the revised manuscript. Furthermore, we will also incorporate the above responses into the revised manuscript to better describe our objectives and the differences of our study from previous studies.

Key references:

Fowler, H.J., Blenkinsop S. and Tebaldi C.: Review: Linking climate change modelling to impacts studies: recent advances in downscaling techniques for hydrological modelling. Int. J. Climatol., 27, 1547-1578, 2007.

Hu, Z., Hu Q., Zhang C., Chen X., and Li Q.: Evolution of reanalysis, spatially interpo-

Printer-friendly version

Discussion paper
Interactive

comment 
lated and satellite remotely sensed precipitation data sets in central Asia. J. Geophys. Res. Atmos., 121, 5648-5663, 2016.

Maraun, D. et al.: Precipitation downscaling under climate change: recent developments to bridge the gap between dynamical models and the end user. Rev. Geophys. 48, RG3003, doi:10.1029/2009RG000314, 2010.

Interactive

comment

Interactive comment on Hydrol. Earth Syst. Sci. Discuss., https://doi.org/10.5194/hess-2017359, 2017. 

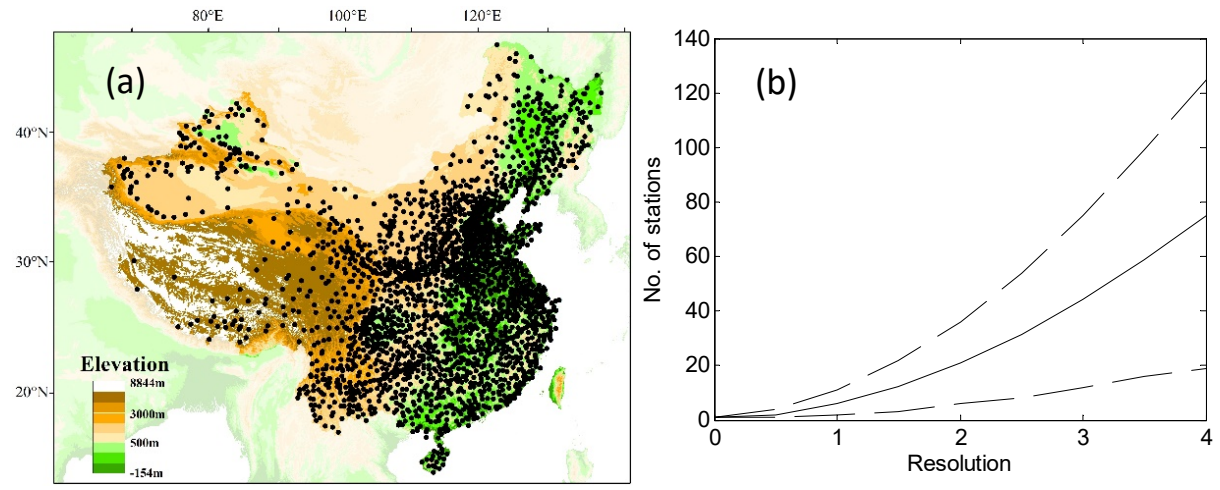

Interactive

comment

Figure R1. Locations of meteorological stations across China (a) and number of stations covered by grid cells with various resolutions centered at each of the stations (b). In (b), the solid line is the averaged number of stations coverved by grid cells centered at the 2,088 stations, the dashed lines are the $10^{\text {th }}-90^{\text {th }}$ percentile. 

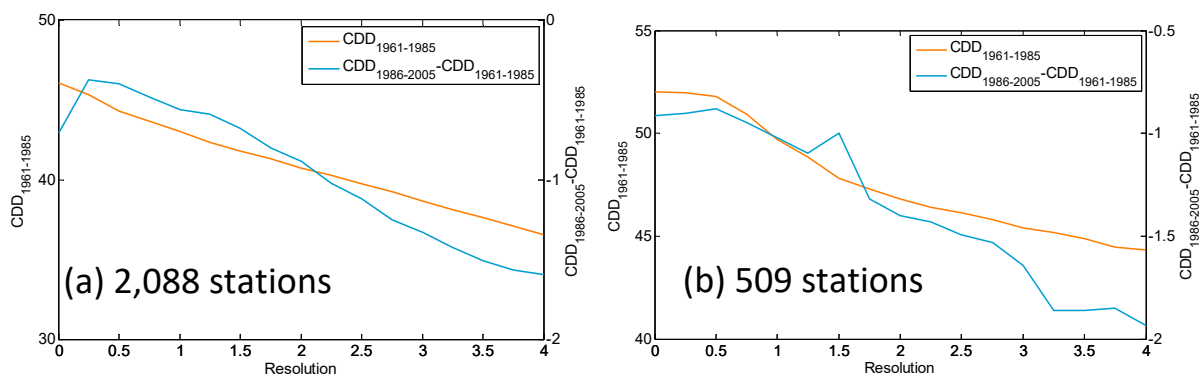

\section{Interactive \\ comment}
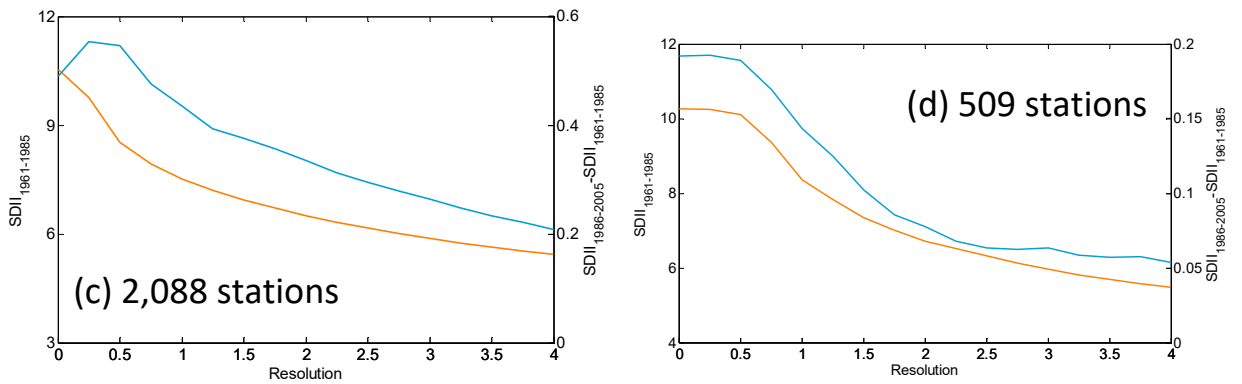

Figure R2. Average magnitudes of 1961-2005 $p_{1961-1985}$ (orange) and changes between 1986-2005 and 1961-1985, i.e. $p_{1986-2005}-p_{1961-1985}$ (blue) of CDD (day) and SDII (mm/day) derived from the 2,088 and 509 stations at various spatial resolutions in degree. 

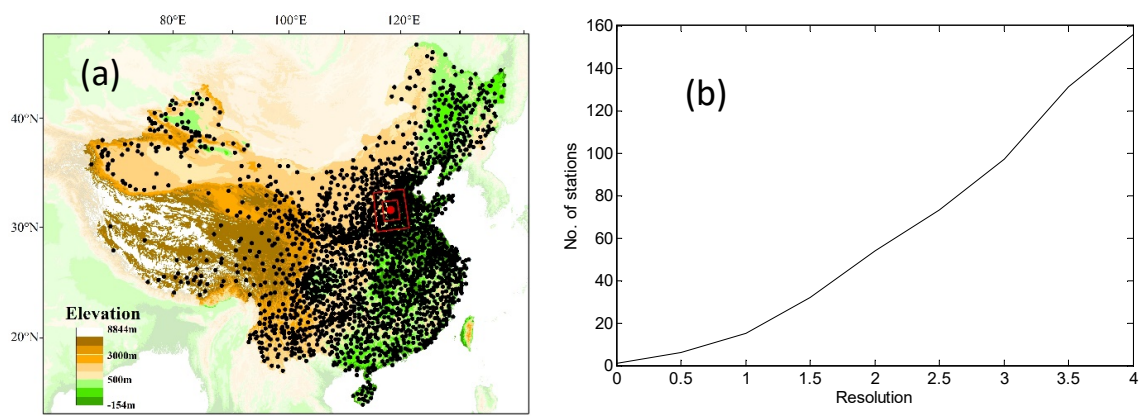

Interactive

comment

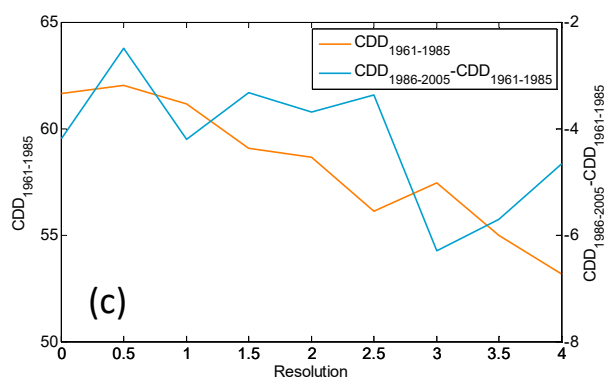

Figure R3. An example of grid cells construction and sensitivity of $\mathrm{CDD}_{1961-1985}$ and $\mathrm{CDD}_{1986-2005}-\mathrm{CDD}_{1961-1985}$ in a station. (a) Grid cells with spatial resolutions of $2^{\circ} \times 2^{\circ}$ (the red inner domain) and $4^{\circ} \times 4^{\circ}$ (the red outer domain) centered at the station (the red point). (b) Number of stations covered by grid cells with various resolutions centered at the station. (c) $C D D_{1961-1985}$ and $C D D_{1986-2005}-C D_{1961-1985}$ estimated by values of grid cells at various resolutions centered at the station. 